Voix et Images

voixetimages

\title{
Le retour de Claude Gauvreau
}

\section{Lucie Robert}

Volume 18, numéro 2 (53), hiver 1993

Francine Noël

URI : https://id.erudit.org/iderudit/201037ar

DOI : https://doi.org/10.7202/201037ar

Aller au sommaire du numéro

\section{Éditeur(s)}

Université du Québec à Montréal

\section{ISSN}

0318-9201 (imprimé)

1705-933X (numérique)

Découvrir la revue

\section{Citer cet article}

Robert, L. (1993). Le retour de Claude Gauvreau. Voix et Images, 18(2), 417-422. https://doi.org/10.7202/201037ar d'utilisation que vous pouvez consulter en ligne.

https://apropos.erudit.org/fr/usagers/politique-dutilisation/ 
Dramaturgie

\section{Le retour de Claude Gauvreau}

\section{Lucie Robert, Université du Québec à Montréal}

Évidemment, mettre en scène une pièce de Claude Gauvreau coûte cher et donne parfois des sueurs froides au producteur du spectacle. Et encore faut-il en trouver qui accepte de courir le risque de présenter cette écriture recherchée, qui nécessite un important déploiement scénique, à un public incertain, rendu maussade par les actuelles difficultés de la vie civile et en particulier par l'imposition d'une TVQ sur le prix des places de théâtre. Pourtant, il y a vingt ans, la création sur une scène professionnelle des textes dramatiques les plus importants de Claude Gauvreau, La Charge de l'orignal épormyable (1970) et Les oranges sont vertes (1972), avait causé tout un émoi et laissé des traces indélébiles dans la mémoire des spectatrices et des spectateurs.

C'est sans doute pour cette raison, c'est-à-dire par un effet de leur mémoire, que, depuis plusieurs années, les critiques de théâtre réclament du Gauvreau (nous réclamons également du Sauvageau, mais ce sera sans doute pour plus tard). Des dix critiques interrogés par Gilbert David qui, en juin 1988, cherchait à constituer un répertoire dramaturgique québécois ${ }^{1}$, la majorité souhaitait en effet revoir sur scène l'un ou l'autre de ces textes. De même et avec raison, je crois, Laurent Mailhot a pu écrire de Claude Gauvreau qu'il était l'un des deux dramaturges vraiment originaux du Québec des années 1950 (l'autre étant Jacques Ferron) ${ }^{2}$. Et pourtant, son œuvre demeure largement méconnue. Quant à savoir laquelle des deux pièces, Les oranges 
sont vertes ou La Charge de l'orignal épormyable, devait être reprise la première, le débat est longtemps resté ouvert. On sait aujourd'hui que La Charge fut élue et produite par le Théâtre de Quat-Sous, à l'automne 1989, dans une mise en scène d'André Brassard. Étonnamment, la seconde production de l'œuvre de Gauvreau ne fut pas l'autre pièce, mais l'adaptation théâtrale de son roman Beauté baroque par Jean Salvy; au Café de la Place des arts, en janvier 1992. Non satisfait de cette leçon de bravoure, Salvy signait récemment une adaptation télévisuelle de La Cbarge de l'orignal épormyable qui fut présentée à Radio-Canada, le 6 décembre dernier, dans le cadre des Beaux Dimanches.

L'événement vaut d'être signalé puisque les œuvres de Claude Gauvreau étaient restées pour ainsi dire inaccessibles au public non spécialisé. En réalité, il n'y a jamais eu d'édition "ordinaire" des textes de cet auteur à la fois poète, romancier et dramaturge. Quelques écrits, surtout les poèmes, avaient été imprimés très discrètement dans les années 1950 et n'avaient pas habité très longtemps les rayons de nos libraires. Sans l'édition des Euvres créatrices complètes ${ }^{3}$, chez Parti pris, en 1977, les trois textes dont il est question ici seraient demeurés dans les tiroirs des héritiers de l'auteur.

À l'époque d'ailleurs, l'édition de Parti pris était apparue comme un événement (Lettres québécoises avait consacré tout un numéro à la lecture des diverses œuvres impliquées), mais qui n'avait eu qu'un succès mitigé, d'une part parce que les "œuvres complètes" n'intéressent en général que quelques spécialistes et que, pour les simples lecteurs, le prix en est souvent bien trop élevé. Ces éditions sont en effet des éditions luxueuses, imprimées sur papier bible, avec signet, etc. Ce qui interdit évidemment aussi d'utiliser l'ouvrage dans. les salles de cours. D'autre part, l'éditeur avait connu ses propres difficultés financières et l'ouvrage avait fait les frais de sa disparition. En réalité, si ma mémoire est bonne, l'édition de ces Euvres créatrices complètes avait été pour le moins épique, entreprise par l'auteur de son vivant (ce qui explique l'existence d'un premier copyright daté de 1971), interrompue par sa mort, retardée par le règlement de succession et réalisée enfin en 1977 (date du dépôt légal). Et j'ignore sans doute les détails les plus juteux de l'affaire. En conséquence, le livre était disparu depuis longtemps des librairies les plus spécialisées et, même d'occasion, il était devenu fort rare. Aussi bien reconnaître encore une fois l'absurdité de la situation éditoriale du Québec qui fait que, fréquemment, les œuvres les plus importantes de la littérature québécoise ne sont disponibles qu'en édition de luxe, livres d'occasion ou microtextes. 
Aussi doit-on souligner l'événement que constitue leur publication par le Groupe Ville-Marie Littérature. D'un côté, VLB publie l'adaptation que Jean Salvy signe de Beauté baroque $e^{4}$ dans sa collection -Théâtre* et, de l'autre côté, les Éditions de l'Hexagone créent une collection des "CEuvres de Claude Gauvreau * qui, pour l'instant, comprend le roman Beauté baroques et le texte de La Charge de l'orignal épormyable ${ }^{6}$. On comprend mal pourquoi l'éditeur a attendu si longtemps pour réaliser cette édition puisque la pièce, reprise il y a deux ans, avait connu un intéressant succès et pas seulement d'estime. Quant à savoir pourquoi on n'a pas du même coup réédité Les oranges sont vertes, vous irez voir vous-mêmes! Souhaitons cependant que la création d'une collection soit aussi une promesse et admettons tout de même - pour la seconde fois en quinze ans -, qu'il était grand temps que l'œuvre de Gauvreau, son œuvre dramatique en particulier, redevienne accessible.

\section{***}

Relire La Charge de l'orignal épormyable n'est pas chose facile. On n'entre pas dans ce texte comme dans n'importe quel autre texte dramatique, souvent réécrit et publié à la hâte, sans que soit véritablement pris en charge le changement de médium. Lire et voir du théâtre sont deux activités différentes et faire passer le texte du théâtre au livre nécessite un travail d'écriture qui comble l'écart. Ce qui étonne d'abord, dans le texte de Gauvreau, c'est l'importance que prend l'écriture. L'auteur raconte autant qu'il montre et dans ses didascalies, il décrit assez longuement pour que la lecture puisse s'effectuer sans trop de mal. Il a le souci de se faire comprendre, ce qui, paraît-il, est passé de mode, mais qui est d'autant plus important dans le présent texte que, sur un autre plan, l'écriture des dialogues porte en elle certaines des expérimentations poétiques de l'auteur. La création de mots nouveaux, tel l'«épormyable du titre accompagne certains usages du langage exploréen. Il y a là uné richesse et une provocation poétique qui ajoutent à l'intérêt dramatique. La difficulté ici n'est donc pas d'ordre technique et elle n'est pas dans l'activité de lecture. Elle est dans ce langage et, encore davantage peut-être, dans le propos riche, dur, sans compromis. Intolérable.

Comment rester froid devant la représentation d'un univers concentrationnaire tel que celui que présente Gauvreau, inspiré de ses séjours à l'hôpital psychiatrique, dit-on, mais qui pourraient tout aussi bien illustrer les expériences "pseudo-scientifiques" réalisées sur les 
animaux de laboratoire (ce que nous rappelle Jacques Godin en guise de postface) ou symboliser un ordre social même pas totalitaire, juste bien pensant. Comment oublier, en fermant le livre, l'image obsédante, bouleversante, d'un Mycroft Mixeudeim qui ne demande qu'à revivre depuis le suicide de celle qu'il aimait, celle de ses bourreaux Lontil-Déparey, Laura $\mathrm{Pa}$, Marie-Jeanne Commode, Becket-Bobo ou Letasse-Cromagon, et celle de Dydrame Daduve qui lui rend un dernier espoir, mais qui sera trompée, elle aussi, comme lui?

Il y a chez Gauvreau cette foi dans la force créatrice de la passion amoureuse authentique, fraîche et parfois un peu naïve, que l'on trouve, mais sur un tout autre registre, dans certains textes de Jean Anouilh et de Jacques Prévert. En même temps, il y a chez lui le refus de toute forme d'espoir, une vision totalement noire et sans avenir de même qu'une représentation sans aucun compromis de la douleur et de la souffrance, à la limite de ce qui est supportable. L'individu reste entièrement écrasé par la société qui l'entoure et qui n'en éprouve pas le moindre regret ni le moindre remords. Les bourreaux ne sont jamais satisfaits et s'acharnent sur les cadavres.

Là est d'ailleurs la principale variante qu'apporte l'adaptation télévisuelle de La Charge de l'orignal épormyable. Pour entrer dans le temps prévu, Salvy a en effet coupé environ un quart du texte de Gauvreau. Dans l'ensemble, ces coupures n'affectent que marginalement le déroulement de la pièce, la concision lui donnant même parfois une plus grande efficacité. Toutefois, les changements de la fin ont une portée plus grande et elles atténuent le pessimisme de l'auteur. D'une part, les "réjouissances" autour du cadavre n'ont pas lieu et il n'est pas dit que le cadavre lui-même va devoir pourrir au milieu de ses bourreaux. D'autre part, la pièce se termine par le magnifique monologue de Mycroft et met mieux en évidence cette phrase que le réalisateur a d'ailleurs reproduite par écrit au début du générique: "Il faut poser des actes d'une si complète audace, que même ceux qui les réprimeront devront admettre qu'un pouce de délivrance a été conquis pour tous."

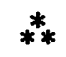

L'adaptation de Beauté baroque, que signe également Jean Salvy, opère à peu près les mêmes transformations du texte original et coupe environ le quart (peut-être un peu plus) du texte. L'opération est plus délicate cependant puisqu'il faut aussi transformer un genre dans un 
autre et faire un dialogue avec un récit. Salvy a tenté de réaliser cette opération sans changer les mots de Gauvreau.

Rappelons que le roman est écrit à la première personne et qu'il se présente comme le récit d'un amour passionné, exclusif et malheureux que le narrateur, un poète, portait à une femme, une comédienne, qui, on l'apprendra à la fin, vient de se suicider. Ce récit se veut également un témoignage, la mémoire des événements et des êtres qui ont conduit la jeune femme au suicide. Le travail d'écriture de Jean Salvy consiste à redistribuer ce récit sur trois voix portées par trois personnages, une femme et deux hommes, le clown noir et le clown blanc, personnages d'un "petit cirque oublié „, habillés de collants de trapézistes, costumes usés, "toujours pauvres", précise le texte. Salvy utilise ainsi l'image - certains diraient le cliché - du clown aux amours malheureuses, dans la plus pure tradition du théâtre populaire français de la fin du siècle dernier, celui qu'a immortalisé Marcel Carné, au grand écran, dans Les Enfants du paradis.

Le problème est que le roman de Gauvreau se distribue mal sur trois voix. Si la voix de la femme peut être construite à partir des dialogues rapportés en style indirect, les voix d'hommes ne font que diluer celle du narrateur-poète. La passion amoureuse qui anime le roman ne peut pas être rendue par deux personnages qui s'observent et se répondent. Elle ne peut non plus être rendue dans le temps présent, un présent continu, qu'impose la forme théâtrale et qui interdit que les événements soient présentés en même temps que leur évaluation dans un regard rétrospectif. Aussi perd-on une partie essentielle de l'œuvre de Gauvreau, l'appel désespéré à la liberté individuelle, la condamnation sans appel de toute forme de répression et la dénonciation de l'univers concentrationnaire. Le social est pour ainsi dire absent du texte de Salvy.

En revanche, on y trouve un grand respect pour la langue de Gauvreau. L'adaptation procède d'un collage de phrases. Si quelquesunes ont été rétablies dans l'ordre syntaxique plus conventionnel qu'exige le texte théâtral, l'ensemble rappelle assez justement la richesse des mots et des images que réinventent constamment les textes de Gauvreau. Au point de laisser croire que c'est précisément cette langue plutôt que le récit qui a séduit Jean Salvy. Et si le social manque au texte c'est peut-être que ce dernier compte trop de belles phrases et que les formules poétiques abondantes y déplacent le propos. L'expérience de cette adaptation valait toutefois d'être tentée, ne fut-ce que pour donner à relire cette œuvre méconnue. Ce que j’ai fait avec le plus grand bonheur. 
L'humeur est parfois mauvaise conseillère, dit-on, et peut-être ai-je eu tort de la laisser déterminer le ton et le contenu de ma dernière chronique. Je me suis laissé entraîner dans l'erreur et j'ai attribué à Alain Fournier le texte de Mon oncle Marcel qui vague vague près du métro Berri ${ }^{7}$, au grand dam de son véritable auteur, Gilbert Dupuis. L'erreur vient du fait que Fournier a signé la mise en scène de la pièce, créée au Théâtre Denise-Pelletier, en mars 1990, et qu'au moment de rédiger ma chronique; travaillant de mémoire et à la dernière minute, c'est ce nom qui m'est venu. La faute n'en est pas moins inexcusable et je comprends fort bien que l'auteur en soit froissé, lui qui a d'ailleurs obtenu le prix du Gouverneur général pour cette pièce. Je ne peux malheureusement pas effacer ce qui a été imprimé et dois me contenter de plates, mais néanmoins sincères excuses. Et maintenant, que celui ou celle qui n'a jamais fait d'erreur me jette la seconde pierre!

1. Gilbert David, :Quelles pièces pour l'an 2000?', Jeu. Cabiers de théâtre, $\mathrm{n}^{\circ} 47$, juin 1988, p. $102-150$.

2. Laurent Mailhot, :D'une histoire dramatique à un espace théâtral., Rerue des sciences bumaines, vol. XIV, $\mathrm{n}^{\circ} 173$, janvier-mars, 1979, p. 91-104.

3. Claude Gauvreau, Guuvres créatrices complètes, édition établie par l'auteur, Montréal, Éditions Parti pris, .Collection du Chien d'Or., 1977, 1498 p. [On trouvera Beauté baroque. Roman moniste, p. 379-500; La Charge de l'orignal épormyable. Fiction dramatique en quatre actes, p. 637-754, et Les oranges sont vertes. Pièce de thêâtre en quatre actes, p. 1363-1488.]

4. Jean Salvy, Beauté baroque, d'après le roman de Claude Gauvreau, Montréal, VLB éditeur, 1992, $123 \mathrm{p}$.

5. Claude Gauvreau, Beauté baroque. Roman moniste, suivi d'une postface de Jean Salvy et des notes d'André G. Bourassa, Montréal, l'Hexagone, coll. . Euuvres de Claude Gauvreau , 1992, 192 p.

6. Id., La Charge de l'orignal épormyable. Fiction dramatique en quatre actes, suivie de témoignages de Pierre Bernard, André Brassard et Jacques Godin, notes d'André-G. Bourassa, Montréal, l'Hexagone, "CEuvres de Ćlaude Gauvreau ", 1992, $250 \mathrm{p}$.

7. Gilbert Dupuis, Mon oncle Marcel qui vague vague près du métro Berri, Montréal, l'Hexagone, coll. “Thêâtre., 1991, 158 p. 